\title{
Chronic Remote Ischemic Conditioning Is Cerebroprotective and Induces Vascular Remodeling in a VCID Model
}

\author{
Mohammad Badruzzaman Khan ${ }^{1} \cdot$ Sherif Hafez $^{1}$ • Md. Nasrul Hoda ${ }^{2}$ Babak Baban ${ }^{3}$. \\ Jesse Wagner ${ }^{1}$ - Mohamed E. Awad ${ }^{3}$ - Hasith Sangabathula ${ }^{1}$ - Stephen Haigh ${ }^{4}$. \\ Mohammed Elsalanty $^{3}$ • Jennifer L. Waller ${ }^{5}$ - David C. Hess ${ }^{1}$
}

Received: 15 May 2017 / Revised: 21 June 2017 / Accepted: 18 July 2017 / Published online: 28 July 2017

(C) The Author(s) 2017. This article is an open access publication

\begin{abstract}
Vascular contributions to cognitive impairment and dementia (VCID) make up 50\% of the cases of dementia. The purpose of this study was to determine the effect of chronic remote ischemic conditioning (C-RIC) on improving longterm (6 months) outcomes and cerebral blood flow (CBF) and collateral formation in a mouse model of VCID. Adult C57BL/6J male mice (10 weeks) were randomly assigned to four different groups: (1) sham-bilateral carotid artery stenosis (BCAS), (2) BCAS + sham RIC, (3) BCAS+C-RIC for 1 month (1MO), and (4) BCAS+C-RIC-4 months (4MO). $\mathrm{CBF}$, cognitive impairment, and functional outcomes were performed up for 6 months after BCAS surgery. The expression of CD31, $\alpha$-SMA, and myelin basic protein (MBP) was assessed by immunohistochemistry (IHC). Additional set of
\end{abstract}

Electronic supplementary material The online version of this article (doi:10.1007/s12975-017-0555-1) contains supplementary material, which is available to authorized users. mice were randomized to sham, BCAS, and BCAS+C-RIC. The cerebrovascular angioarchitecture was studied with micro-CT. RIC therapy for either 1 or 4 months significantly improved CBF, new collateral formation, functional and cognitive outcomes, and prevented white matter damage. There was no difference between C-RIC for 1 or 4 months; IHC studies at 6 months showed an increase in brain CD31 and $\alpha$-SMA expression indicating increased angiogenesis and MBP indicating preservation of white matter in animals receiving RIC. One month of daily RIC is as effective as 4 months of daily RIC in improving CBF, angiogenesis, and long-term functional outcomes (6 months) in a VCID model. This suggests that 1 month of RIC is sufficient to reduce cognitive impairment and induce beneficial cerebrovascular remodeling.

Keywords Chronic remote ischemic conditioning (C-RIC) · Cerebral blood flow (CBF) - Vascular contributions to cognitive impairment and dementia (VCID) - Angiogenesis, collateral remodeling, white matter degeneration

$\begin{array}{ll}\text { Abbreviations } \\ \text { 1MO } & \text { One month } \\ \text { 4MO } & \text { Four months } \\ 6 \mathrm{MO} & \text { Six months } \\ \text { BCAS } & \text { Bilateral carotid artery stenosis } \\ \text { CCAs } & \text { Common carotid arteries } \\ \text { CBF } & \text { Cerebral blood flow } \\ \text { RIC } & \text { Remote ischemic conditioning } \\ \text { C- } & \text { Chronic remote ischemic conditioning } \\ \text { RIC } & \\ \text { GFAP } & \text { Glial fibrillary acidic protein } \\ \text { IF } & \text { Immunofluorescence } \\ \text { IHC } & \text { Immunohistochemistry }\end{array}$




$\begin{array}{ll}\text { LSCI } & \text { Laser Speckle Contrast Imager } \\ \text { MBP } & \text { Myelin basic protein } \\ \text { NO } & \text { Nitric oxide } \\ \text { VCID } & \begin{array}{l}\text { Vascular contributions to cognitive impairment and } \\ \text { dementia }\end{array} \\ \text { WM } & \text { White matter }\end{array}$

\section{Introduction}

The prevalence of dementia is expected to triple by 2050, a major threat to the world's public health. Vascular dementia makes up to $20 \%$ of the cases of dementia, and mixed dementia (vascular and Alzheimer's) is estimated to be up to $50 \%$ of the cases of dementia [1]. Moreover, cerebral ischemia worsens Alzheimer's disease (AD) and triggers its clinical expression [2]. Vascular contributions to cognitive impairment and dementia (VCID) is a term that encompasses the vascular factors and vascular pathology that underlie the clinical spectrum from mild cognitive impairment to dementia. VCID is a major research focus of the National Institute of Neurological Diseases and Stroke [3, 4]. Other than controlling the comorbid vascular risk factors, there is no known effective treatment for VCID. However, clinical observational studies strongly suggest that physical exercise is effective at reducing progression of cognitive decline and dementia [5].

Reduction of cerebral blood flow (CBF) may be the key precipitating event in $\mathrm{AD}$ and VCID [1]. Hypoperfusion appears to be an early finding that plays a pathophysiological role in the development of white matter (WM) damage [6]. Low blood flow by MRI perfusion or MRI arterial spin labeling (ASL) is predictive of white matter lesions [7, 8]. A penumbra exists around white matter lesions that expand in relation to low CBF [8]. An animal model for VCID is essential to test new interventions.

In a recent review of all mouse models for VCID, Bink et al. determined the mouse bilateral carotid artery stenosis (BCAS) model to be the most valid [9]. This model reproduces the WM damage, cerebral hypoperfusion, inflammation, BBB damage, and cognitive deficits of the human condition $[10,11]$. There are also fibrinoid changes in the small vessels of the brain with gliosis and disruption of aquaporin polarization [12]. With these small vessel changes, the BCAS model may be useful to test interventions to treat small vessel disease of the brain [12].

Remote limb ischemic conditioning (RIC) is the simple, inexpensive, and safe use of repetitive inflation of a blood pressure (BP) cuff on the arm or leg to protect distant organs such as the brain from ischemic injury. Chronic RIC (C-RIC) is the repetitive use of daily RIC for periods of weeks or months. RIC shares common mechanisms with physical exercise and may be viewed as an "exercise equivalent" $[13,14]$. We previously showed in a BCAS mouse model that C-RIC for 2 weeks increased $\mathrm{CBF}$ in a sustained fashion, reduced WM damage, improved cognitive performance, and reduced accumulation of amyloid-beta 42 protein $\left(A \beta_{42}\right)$ in the brain [15].

However, whether RIC can induce long-term neuroprotection and how long RIC would need to be applied are not known. This study aimed to determine if C-RIC can induce long-term (at 6 months) neurovascular protection, and if 1 month of daily RIC is as effective as 4 months in a BCAS model. Our secondary aim was to determine whether C-RICinduced neuroprotection is attributed to RIC-induced vascular remodeling and angiogenesis in the brain, similar to what is seen with chronic physical exercise.

\section{Materials and Methods}

\section{Animal Models and Experimental Groups}

The animals were housed at Augusta University's animal care facility, which is approved by the American Association for Accreditation of Laboratory Animal Care. This study was conducted in accordance with the National Institute of Health guidelines for the care and use of animals in research, and all protocols were approved by the institutional animal care and use committee. All the STAIR (Stroke Therapy Academic Industry Roundtable) and RIGOR recommendations and guidelines regarding randomization, blinding, and statistical analysis were followed in this study [16, 17].

\section{Determine the Effect of Chronic RIC on Cognitive Impairment, Functional Outcomes, and CBF}

Forty male mice were randomized into four groups: (1) sham (operated group for procedures of BCAS), (2) BCAS and sham RIC, (3) BCAS treated with daily RIC for 1 month post BCAS surgery (BCAS + RIC-1MO, $N=10$ ), and (4) BCAS treated with daily RIC for 4 months post BCAS surgery (BCAS + RIC-4MO, $N=10$ ). The outcomes were assessed by a blinded investigator. Cognitive function, functional outcomes, and $\mathrm{CBF}$ changes were considered as the primary outcomes and were determined at 4 and 6 months after BCAS, with either $1 \mathrm{MO}$ or $4 \mathrm{MO}$ RIC. Thereafter, the animals were sacrificed, the blood was collected for plasma nitrite estimation, and the brains were isolated and dissected for IHC (Schematic representation of the study plan in Supplemental Fig. 1).

\section{Bilateral Carotid Artery Stenosis Surgical Procedure}

BCAS was performed as previously described $[10,15]$. In brief, animals were anesthetized with $2 \%$ isofluorane and both common carotid arteries (CCAs) were exposed by a midline 
cervical incision. Customized microcoils, specially designed to mimic a VCID model in the mice which was made of steel wire with an inner diameter of $0.18 \mathrm{~mm}$, was twined by rotating around both right and left CCA at 30 min interval.

\section{Non-Invasive RIC Therapeutic Methods}

Non-invasive RIC therapy method was performed as published elsewhere $[18,19]$ with a programmable cuff or its sham procedure with a cuff that did not inflate or deflate (see detail in "Supplemental Methods") [15]. Bilateral RIC therapy was performed in both hind limbs simultaneously $(4$ cycles $\times 5$ min duration of each cycle inflate and deflate $\times$ 5 min interval between each cycle) daily for 1 month (BCAS+ RIC-1MO) or 4 months (BCAS+RIC-4MO) after 1 week from BCAS surgery.

\section{Cerebral Blood Flow by Laser Speckle Contrast Imager}

Cerebral blood flow (CBF) was measured using highresolution Laser Speckle Contrast Imager (LSCI) (PSI system, Perimed Inc.) at different time points as indicated in the figure and as previously reported by us [15]. Mice were placed on a warming pad and thermostatically controlled at around $37^{\circ} \mathrm{C}$ to avoid the effect of body temperature during the measurement of CBF.

\section{Estimation of Nitrite in Plasma}

Plasma $\mathrm{NO}_{x}\left(\mathrm{NO}_{2}^{-}+\mathrm{NO}_{3}^{-}\right)$levels were measured by NOspecific chemiluminescence, as described previously [20] with slight modification. Briefly, 100 ul of plasma were mixed with twice volumes of ethanol (100\%) and kept at $-20 \mathrm{C}$ for 40-60 min followed by centrifuged at $13000 \mathrm{rpm}$ for $10 \mathrm{~min}$ to remove protein as pellet. The supernatant $(100 \mathrm{ul})$ was taken and injected to measure nitrite. The nitrite levels were measured by NO Analyzer 280i (GE Analytical Instruments, CO, USA). The level of nitrite was expressed in nanomolar.

\section{Behavioral Test: Functional Outcomes and Cognitive Test}

An investigator who was blinded to the experimental design and behavioral test or treatment including NOR test, [21] Ymaze test, [22] beam walk, [23] and wire hanging test [24] (Detailed procedures are explained in the "Supplemental Methods."). All groups were examined at 4 and 6 months after sham surgery, BCAS surgery, and with RIC (1MO or 4MO). NOR and Y-maze test were performed for cognitive test where beam walk test required mice to balance on a wooden beam to evaluate any gait abnormality and wire hanging test for muscular or motor function.

\section{Histological and Immunohistochemical Assessment}

Immunohistochemistry was performed according to the protocol previously described [15] with slight modification. Both paraffin and cryo-sections were used for immunohistochemistry and immunofluorescence with similar anatomic features (see the "Supplemental Methods" for details). The primary antibodies were anti-myelin basic protein (MBP) (C-16 clone, SC-13914, Santa Cruz, CA, USA; 1:100 dilution); rat anti-platelet endothelial cell adhesion molecule [PECAME-1 (CD31), BD no. 550274, USA,1:200 dilution], with mouse monoclonal anti- $\alpha$-smooth muscle actin $[\alpha$-SMA, SC-53142, Santa Cruz, CA, USA; 1:50 dilution]; and anti-plateletderived growth factor [PDGFR- $\beta$ (958): SC-432, Santa Cruz, CA, USA; 1:200 dilution], isolectin B4 conjugates (Invitrogen, molecular probe Life Technology, IB4 no. 121411). For biotinylated immunostaining, the brain sections were incubated in the anti-MBP primary antibodies and using the avidin-biotin-peroxidase complex method with diaminobenzidine (DAB) as the chromogen. The immunostaining was carried out using the $\mathrm{ABC}$ kit system (Vector, Burlingame, CA, USA). After staining, the sections were counterstained with Harris hematoxylin (cat. no. HHS35-1L; Sigma) for few seconds. The sections were then dehydrated rapidly through ethanol and xylene and mounted with VectaMount medium (Vector).

\section{Assessment of New Collateral Formation and Angiogenesis: Micro-CT and BriteVu Methods}

Twenty-seven male mice were randomized into three groups: (1) sham, (2) BCAS and sham RIC (BCAS), and (3) BCAS treated with daily RIC for 3 weeks post BCAS surgery (BCAS+RIC). Three weeks after BCAS, blood was collected either from the eye (retro orbital) for flow cytometry analysis of endothelial progenitor cells (EPCs) and macrophages, or from the heart plasma nitrite estimation. Animals were transcardially perfused with heparinized saline to flush out the blood, followed

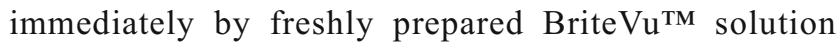
(Scarlet Imaging, LCC; Murray, UT, USA) according to manufacturer instruction. Carcasses were kept on ice at $4{ }^{\circ} \mathrm{C}$ overnight to ensure solidification of BriteVu dye prior to imaging. After $24 \mathrm{~h}$, brains were isolated, fixed with $4 \%$ PFA for $48 \mathrm{~h}$, and then saved in $70 \%$ alcohol till the time of imaging. SkyScan 1174 (Bruker micro$\mathrm{CT}$ /formerly known as SkyScan, USA) is used for imaging. SkyScan is associated with a full-range software that provides fast volumetric reconstruction of $2 \mathrm{D} / 3 \mathrm{D}$ quantitative analysis and realistic $3 \mathrm{D}$ visualization of cerebrovasculature. Outcomes: (1) full 3D picture of 
a

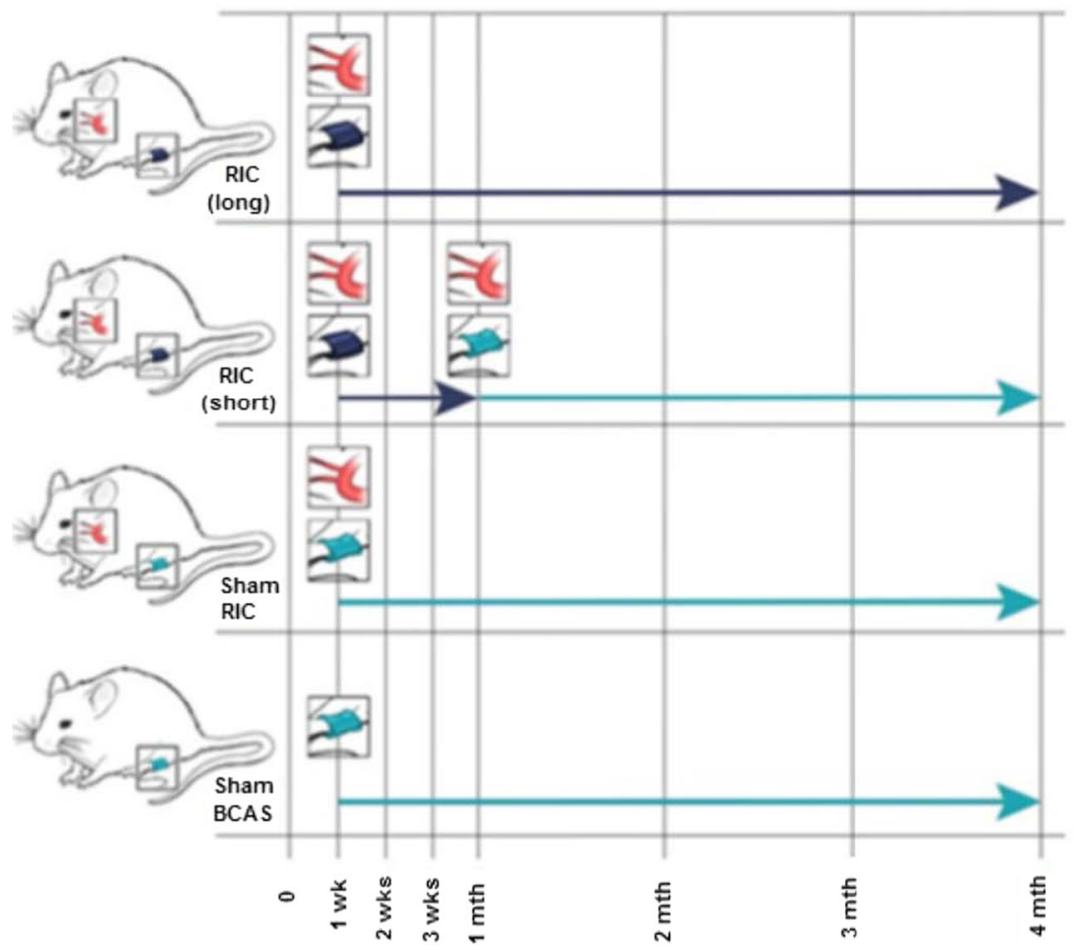

CBF at 4 months
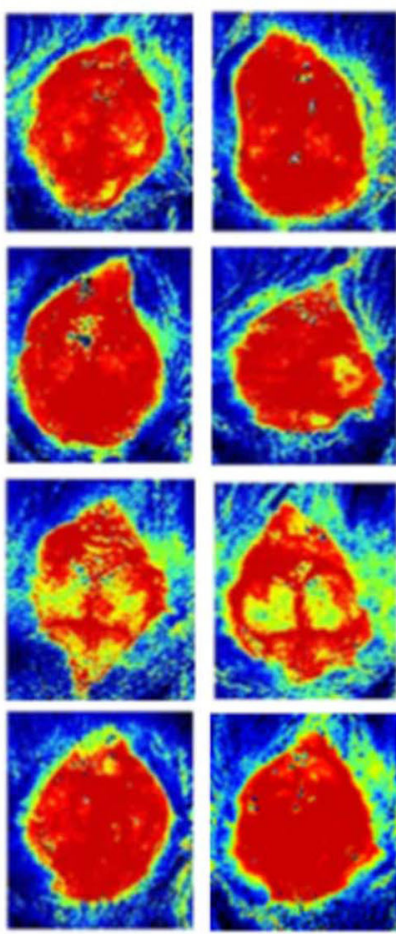
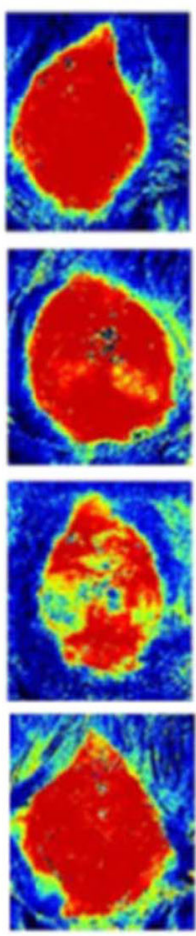

b

CBF pre BCAS surgery

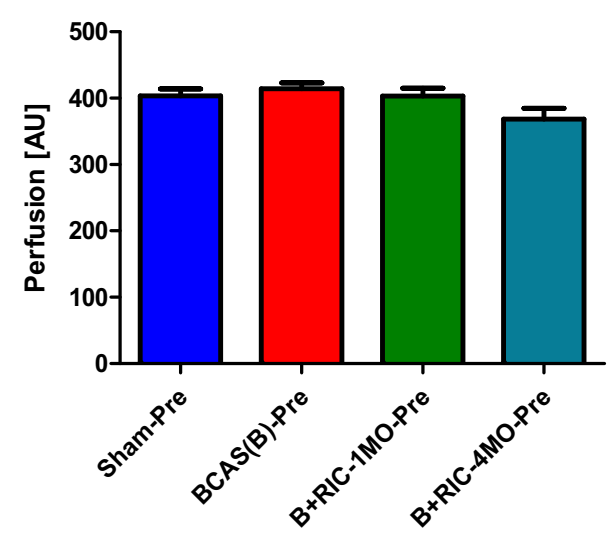

d $\mathrm{CBF}$ at 4 months post RIC or sham RIC

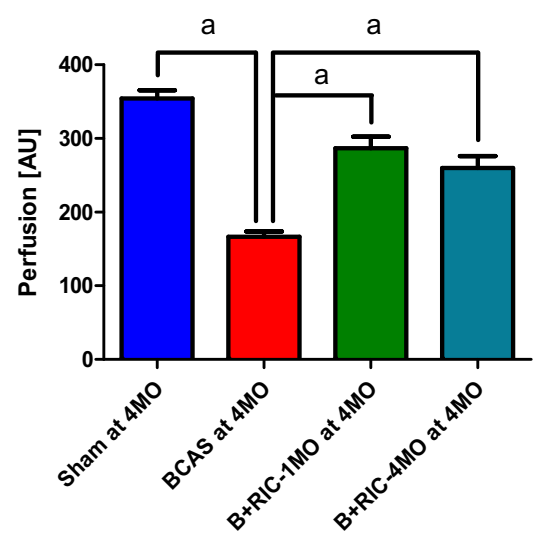

C CBF at post BCAS or sham BCAS

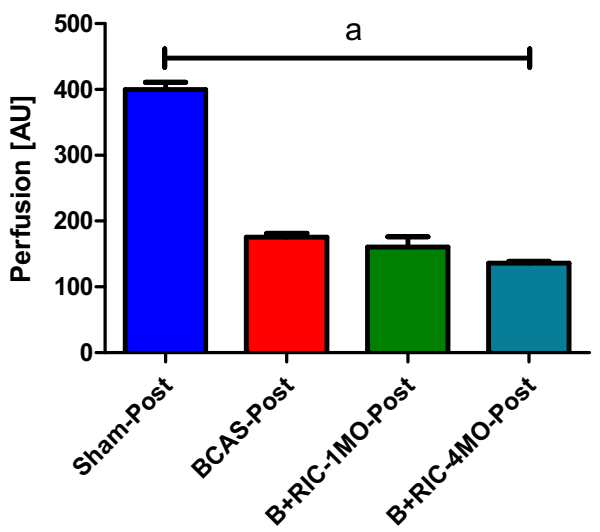

e CBF at 6 months post RIC or Sham RIC
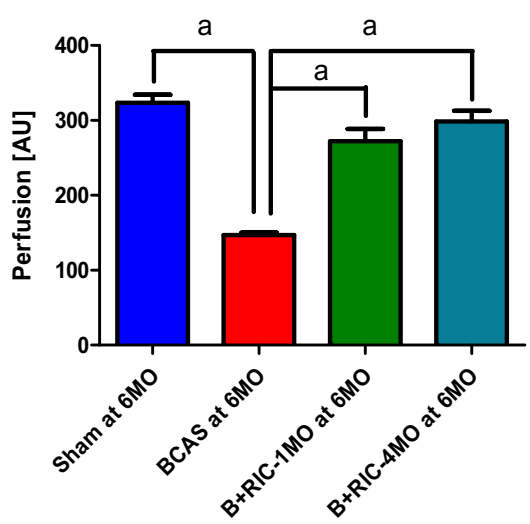
Fig. 1 Measuring of $\mathrm{CBF}$ changes by Laser Speckle Contrast Imager (LSCI), remote ischemic conditioning (RIC) (1-MO and 4-MO therapy at $4 \mathrm{MO}$ and $6 \mathrm{MO})$ increases cerebral blood flow $(\mathrm{CBF})$ in bilateral carotid artery stenosis (BCAS) mice. a Mice underwent BCAS and were randomized to RIC daily for 4 months (long with dark blue arrow, top row), RIC daily for 1 month (short with dark blue arrow, second row), or sham RIC (green arrow, third row). The bottom row shows mice with sham BCAS surgery (no coils). CBF was measured at 4 and 6 months (Supplemental Fig. 2) in all mice. Daily RIC for 1 month produced similar increases in CBF to daily RIC for 4 months. Red indicates higher blood flow. $\mathbf{b}-\mathbf{e}$ Absolute value of cerebral perfusion in perfusion unit (PU) at different time points where pre (prior to BCAS), post (after BCAS and prior to RIC), at 4 months (4MO) and at 6 months (6MO); pre and post sham operation, BCAS with sham RIC; and BCAS+RIC with $1 \mathrm{MO}$ and $4 \mathrm{MO}$. Using repeated measures mixed models, there is no significant difference between $\mathrm{CBF}$ in the $1 \mathrm{MO}$ and $4 \mathrm{MO}$ therapy groups but both groups are significantly higher than the BCAS sham group at $4 \mathrm{MO}$ and at $6 \mathrm{MO}\left(N=7\right.$ to $10 /$ groups; ${ }^{\mathrm{a}} p<0.0001$ vs BCAS+sham-RIC; ${ }^{\mathrm{a}} p<0.0001$ vs BCAS+RIC-1MO and BCAS+RIC-4MO)

cerebrovasculature and (2) quantification of vascular number, vascular volume, vascular density, lumen diameter, and formation of new collaterals.

\section{Evaluation of EPCs and Macrophages}

Whole blood (WB) was collected using heparinated microtubes as described previously with slight modification $[25,26]$. One hundred fifty milliliters of WB were then incubated with antibodies for EPC markers including CD31, CD34, VEGFR2, and surface markers for M1/M2 macrophages, CD11b, F4/80, and CD206 (eBioscience, USA) for 20 min on ice in the dark. After washing was completed, cells were fixed and permeabilized using fix/perm concentrate (eBioscience, USA) before incubation with antibodies for intracellular staining of TNF $\alpha$ (for M1 macrophages) and IL10 (for M2 macrophages). Samples were then washed and run through a four-color flow cytometer (FACSCalibur, BD Biosciences), and data were collected using the CellQuest software. Samples were double-stained with control $\mathrm{IgG}$ and cell markers to assess any spillover signal of fluorochromes. Proper compensation was set to ensure that the median fluorescence intensities of negative and positive cells were identical and then was used to gate the population. Gating excluded dead cells and debris using forward and side scatter plots. To confirm the specificity of primary antibody binding and rule out non-specific $\mathrm{Fc}$ receptor binding to cells or other cellular protein interactions, negative control experiments were conducted using isotype controls matched to each primary antibody's host species, isotype, and conjugation format.

\section{Statistical Analysis}

All statistical analysis was performed using SAS 9.4, and statistical significance was assessed using a significance level of 0.05 . To examine differences between the groups (sham, BCAS, BCAS+RIC-1MO, BCAS+RIC$4 \mathrm{MO}$ ) for nitrites, a one-way ANOVA was used. If the overall test for the one-way ANOVA was statistically significant a Tukey-Kramer multiple comparison procedure was used to examine differences between the four groups. For cerebral blood flow, beam walk, hanging wire, NOR, and Y-maze measures, a repeated measures mixed model was used to examine differences between the four groups over time ( $\mathrm{CBF}$ measurement times: baseline, post surgery, 4 months, 6 months; all other outcomes measurement times: 4 months, 6 months). Main effects of group and time as well as the twofactor interaction between group and time were included in the model. For CBF, an auto-regressive order 1 correlation structure fit the data best and was used to account for the correlation between measurement times. For the beam walk, hanging wire, NOR discrimination index, and Y-maze measures, an unstructured correlation structure was used as there were only two measurement times. Of statistical interest was the F-test for the twofactor interaction between group and time, and if it is statistically significant, it indicates that the change in the outcome over time is different for the four groups. To examine differences between groups within measurement time and between measurement times within group, a Bonferroni adjustment to the overall alpha level was used to control for the multiple post hoc pairwise tests as not all pair-wise tests were of interest. For $\mathrm{CBF}$, the Bonferroni adjusted alpha is 0.0010 and for the other outcomes, the Bonferroni adjusted alpha is 0.0031 .

\section{Results}

\section{RIC Improved Cerebral Blood Flow}

C-RIC therapy after BCAS increased CBF compared to sham RIC (Fig. 1a-e; Supplemental Fig. 2). CBF was significantly increased in both $1 \mathrm{MO}$ and 4MO RIC therapy as compared to BCAS-sham RIC. Moreover, $1 \mathrm{MO}$ of RIC was as effective as 4MO RIC in improving CBF at 6 months.

\section{RIC Improved Cognition Through Enhanced Spatial and Working Memory}

Both $1 \mathrm{MO}$ and 4MO RIC improved cognition. Results from NOR test (for spatial memory) (Fig. 2a-d) and Y-maze test for working memory (Supplemental Fig. 3A-D) showed that the sham group is more attracted toward novel objects compared to BCAS. The BCAS group had significantly shorter exploration time for novel object (TN) and less discrimination index (DI). This indicates that BCAS causes impairment of 
a Exploration time, TN at 4 mo after BCAS

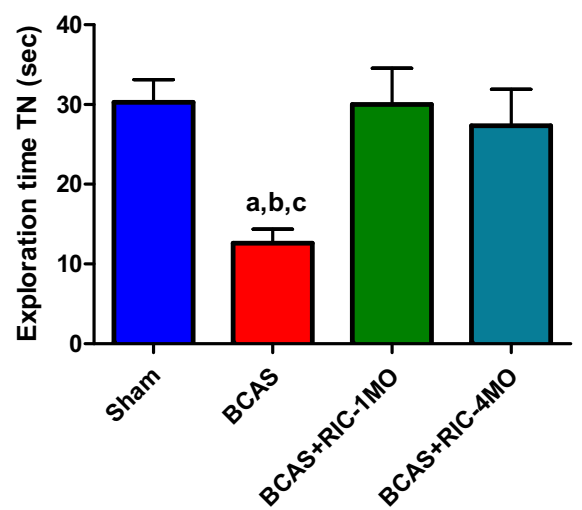

C Discrimination index (DI) at 4 month after BCAS

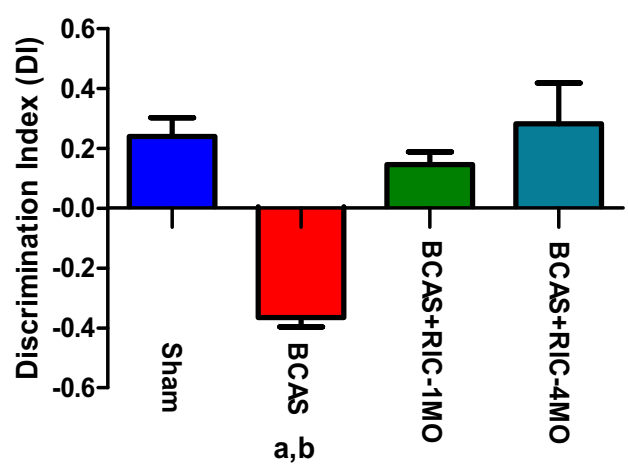

Fig. 2 Using a repeated measures mixed model for time of exploration across (a) and (b),(a) time of exploration (TN) spent with the novel object at 4 months (4MO) after sham operation, BCAS, and BCAS+RIC with $1 \mathrm{MO}$ and $4 \mathrm{MO}$. The RIC for $1 \mathrm{MO}$ and $4 \mathrm{MO}$ groups shows no significant difference but is significantly different than BCAS (sham RIC). Values are indicated as mean \pm SE. ${ }^{a} p<0.001$ vs sham; ${ }^{b} p<0.001$ vs BCAS+ RIC-1MO; ${ }^{c} p<0.01$ vs BCAS+RIC-4MO. (b) TN spent with the novel object at 6 months $(6 \mathrm{MO})$ after sham operation, BCAS, and BCAS+RIC with $1 \mathrm{MO}$ and $4 \mathrm{MO}$. Values are indicated as mean \pm SE. ${ }^{\mathrm{a}} p<0.001 \mathrm{vs}$ sham; ${ }^{\mathrm{b}} p<0.001$ vs BCAS+RIC-1MO; ${ }^{\mathrm{c}} p<0.01$ vs BCAS+RIC-4MO. Using a repeated measures mixed model for the discrimination index

discriminative ability in mice. RIC therapy for either 1-MO or 4-MO significantly restored the TN and DI scores. Moreover, RIC therapy for either $1 \mathrm{MO}$ or 4-MO significantly increased the entries' alternations in the arms of Y-maze compared to the sham group tested at 4 and 6 months after BCAS.

\section{RIC Improved Muscular/Motor Function}

Animals subjected to BCAS spent more time to cross a beam in comparison to the sham control animals (Fig. $3 \mathrm{a}, \mathrm{b})$. RIC therapy for either $1 \mathrm{MO}$ or $4 \mathrm{MO}$ significantly reduced the time spent by the animals to cross the beam as compared to BCAS groups. Moreover, muscular impairment or motor impairment was assayed by wire hanging test (Fig. 3c, d). BCAS groups spent b Exploration time, TN at 6 mo after BCAS

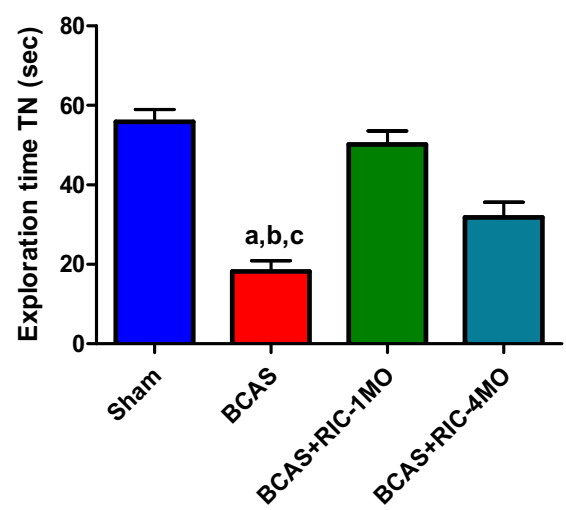

d Discrimination index (DI) at 6 month

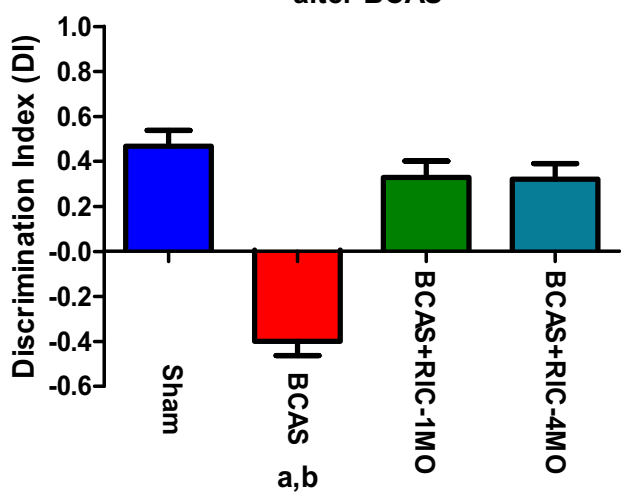

across (c) and (d), (c) the discrimination index (DI) at 4 months (4MO) after sham operation, BCAS, and BCAS+RIC with $1 \mathrm{MO}$ and 4MO.The BCAS+RIC with $1 \mathrm{MO}$ and $4 \mathrm{MO}$ groups show no significant difference from one another but both are significantly different from the BCAS sham RIC. Values are indicated as mean \pm SE. ${ }^{a} p<0.0001$ vs sham; ${ }^{b} p<0.0001$ vs BCAS+RIC-1MO and BCAS+RIC-4MO. (d) The discrimination index (DI) at 6 months (6MO) after sham operation, BCAS, and BCAS+ RIC with $1 \mathrm{MO}$ and $4 \mathrm{MO}$. There is no difference between $1 \mathrm{MO}$ and $4 \mathrm{MO}$ RIC groups, but both are significantly different than BCAS (sham RIC). Values are indicated as mean \pm SE. ${ }^{\mathrm{a}} p<0.0001 \mathrm{vs}$ sham; ${ }^{\mathrm{b}} p<0.0001 \mathrm{vs}$ BCAS+RIC-1MO and BCAS+RIC-4MO

significantly less time suspending their body to the hanging wire compared to sham. RIC therapy for $1 \mathrm{MO}$ or $4 \mathrm{MO}$ significantly increased the hanging wire time compared to the BCAS group.

\section{RIC Enhanced Angiogenesis and Arteriogenesis}

RIC therapy significantly increased capillary density, angiogenesis, and arteriogenesis as indicated by increased expression of CD31 and $\alpha$-SMA, compared to the BCAS group (Fig. 4a-d; Supplemental Figs. 4 and 5). However, there was no significant difference between RIC-1MO and RIC-4MO therapy groups. Moreover, RIC therapy increased the expression and colocalization of pericytes with cerebral blood vessels as indicated by 
a Beam walk crossing test at 4 months

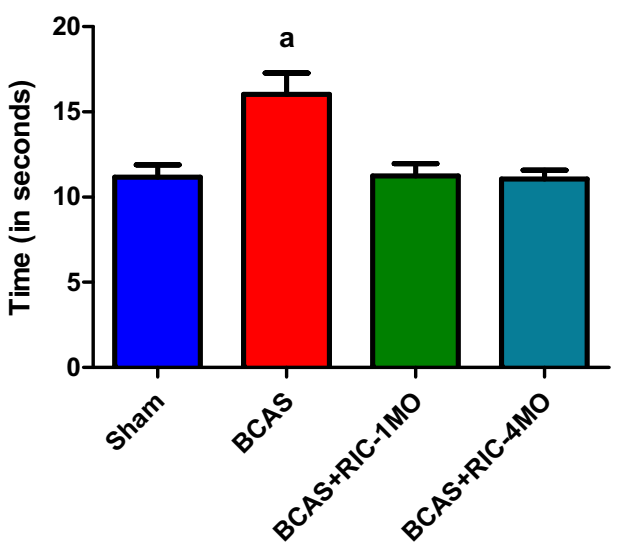

C Hanging wire test at 4 months

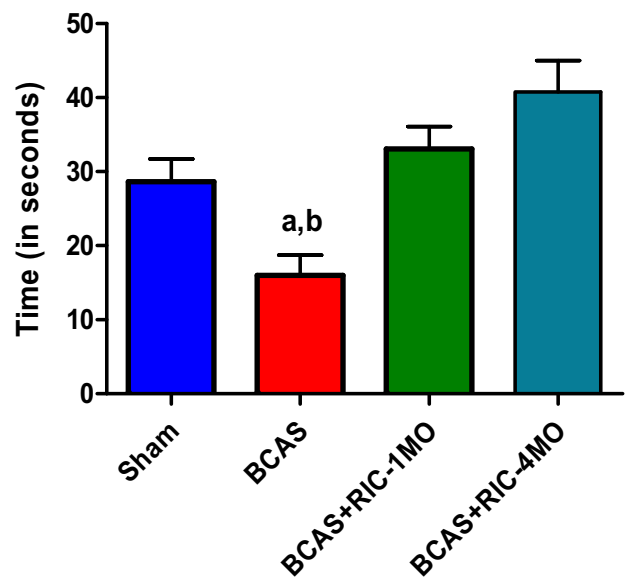

Fig. 3 RIC prevents motor/muscular impairment after BCAS. Using a repeated measures mixed model for beam walk across (a) and (b), (a) time of crossing on a beam at 4 months (4MO) after sham operation, BCAS, and BCAS+RIC with $1 \mathrm{MO}$ and $4 \mathrm{MO}$. Values are indicated as mean \pm SE. ${ }^{a} p<0.0001$ vs sham, BCAS+RIC-1MO, and BCAS+RIC$4 \mathrm{MO}$. (b) Time of crossing on a beam at 6 months (6MO) after sham operation, BCAS, and BCAS+RIC with $1 \mathrm{MO}$ and $4 \mathrm{MO}$. Values are indicated as mean \pm SE. ${ }^{\mathrm{a}} p<0.0001$ vs sham, BCAS+RIC-1MO, and BCAS+RIC-4MO. Using a repeated measures mixed model for the hanging wire test across (c) and (d), (c) cord-wire hanging test for muscular

increased expression of PDGFR-B and IB4, respectively (Supplemental Fig. 6A). Chronic treatment also showed to increase the capillary diameter (Supplemental Fig. 6B).

\section{RIC Reduced White Matter Damage and Myelin Basic Protein (MBP)}

The white matter degeneration in the corpus callosum was tested by Klüver-Barrera staining. The intensity in KlüverBarrera staining was significantly reduced after BCAS compared to sham animals. RIC therapy for 1 or 4 months significantly reversed the BCAS-induced white matter damage (Supplemental Fig. 7A-B). MBP staining significantly decreased in cortical and hippocampal CA1 field region

\section{b Beam walk crossing test at 6 months}
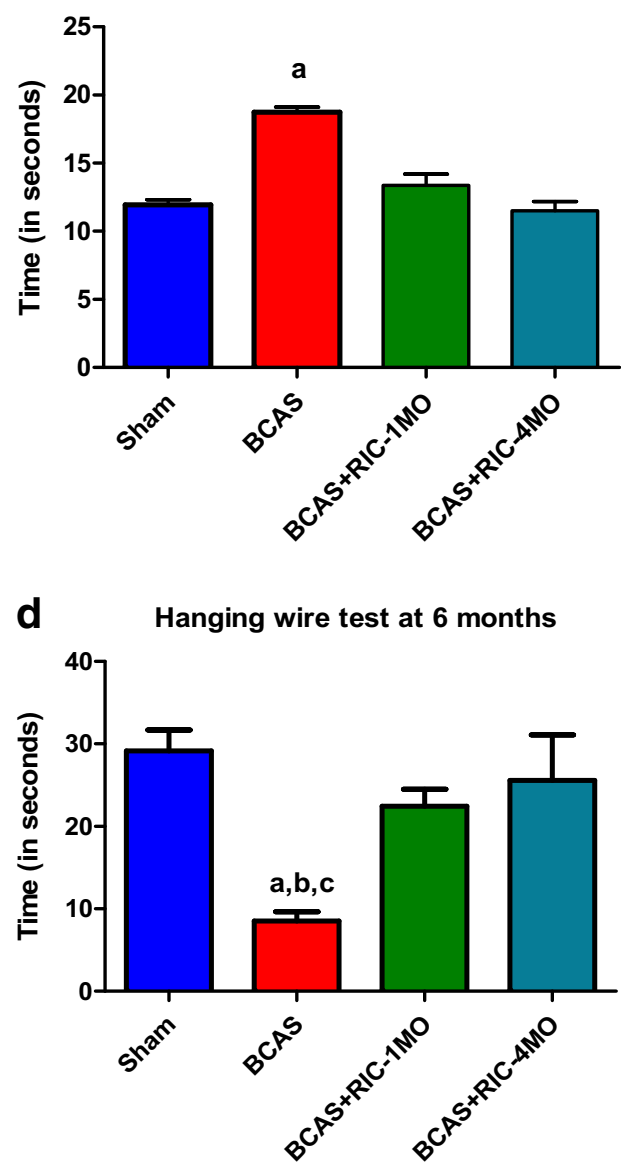

impairment at 4 months (4MO) after sham operation, BCAS, and BCAS+ RIC with $1 \mathrm{MO}$ and $4 \mathrm{MO}$. Values are indicated as mean \pm SE. ${ }^{\mathrm{a}} p=0.0008$ vs sham; ${ }^{\mathrm{b}} p<0.0001$ vs BCAS+RIC-1MO and BCAS+RIC-4MO. (d) Cord-wire hanging test for muscular impairment at 6 months (4MO) after sham operation, BCAS, and BCAS+RIC with $1 \mathrm{MO}$ and $4 \mathrm{MO}$. There is no difference between $1 \mathrm{MO}$ and $4 \mathrm{MO}$ RIC groups, but both significantly are different than BCAS (sham RIC). Values are indicated as mean $\pm \mathrm{SE}$. ${ }^{\mathrm{a}} p<0.0001$ vs sham; ${ }^{\mathrm{b}} p=0.0008$ vs BCAS+RIC- $1 \mathrm{MO} ;{ }^{\mathrm{c}} p=0.0001$ BCAS+RIC-4MO

(Supplemental Fig. 8A-B), and this was reversed with RIC therapy in both BCAS+RIC1-MO and BCAS+RIC4-MO groups.

\section{RIC Improves the Cerebrovascular Angioarchitecture and Increases NO Production}

The use of BriteVu staining enabled the $3 \mathrm{D}$ visualization of the complete tree of the cerebrovasculature. BCAS caused a significant decline in number and volume of cerebral vessels. RIC significantly induced angiogenesis and collaterals formation as indicated by the increase in the vessels number and volume (Fig. 5a-c; Supplemental Fig. 9A-C). In support with this, results 

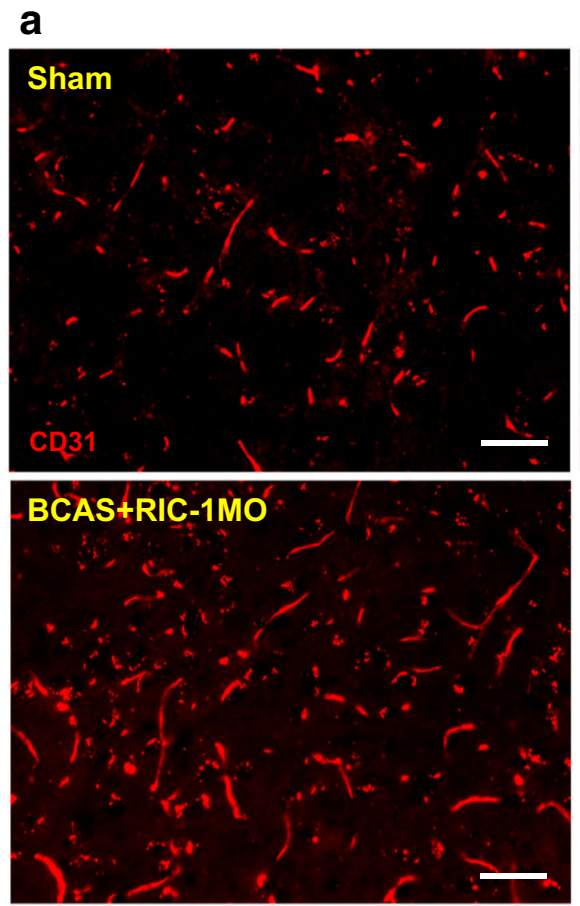

C
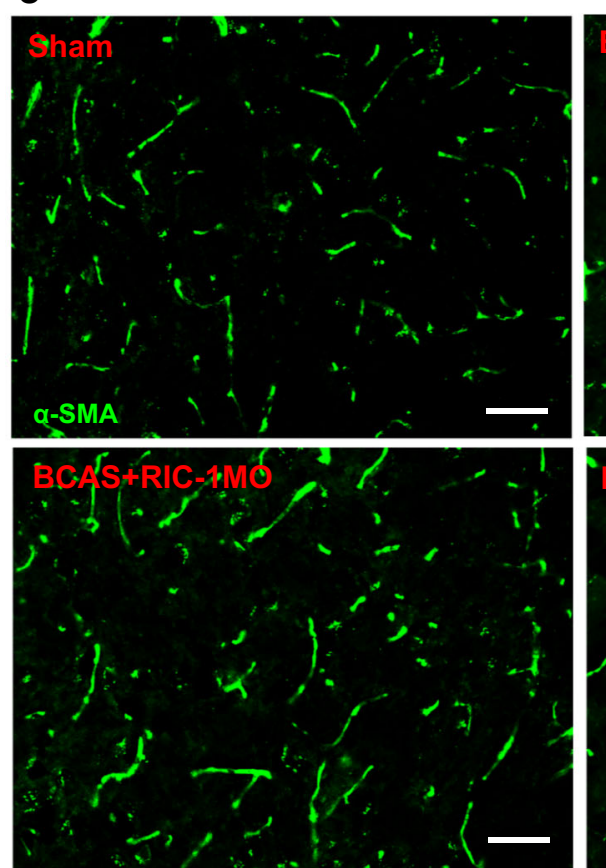

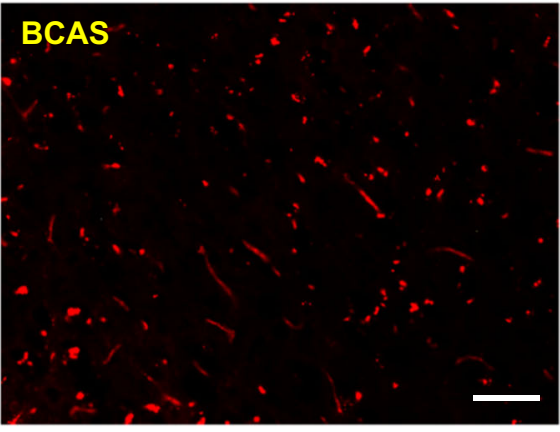

BCAS+RIC-4MO
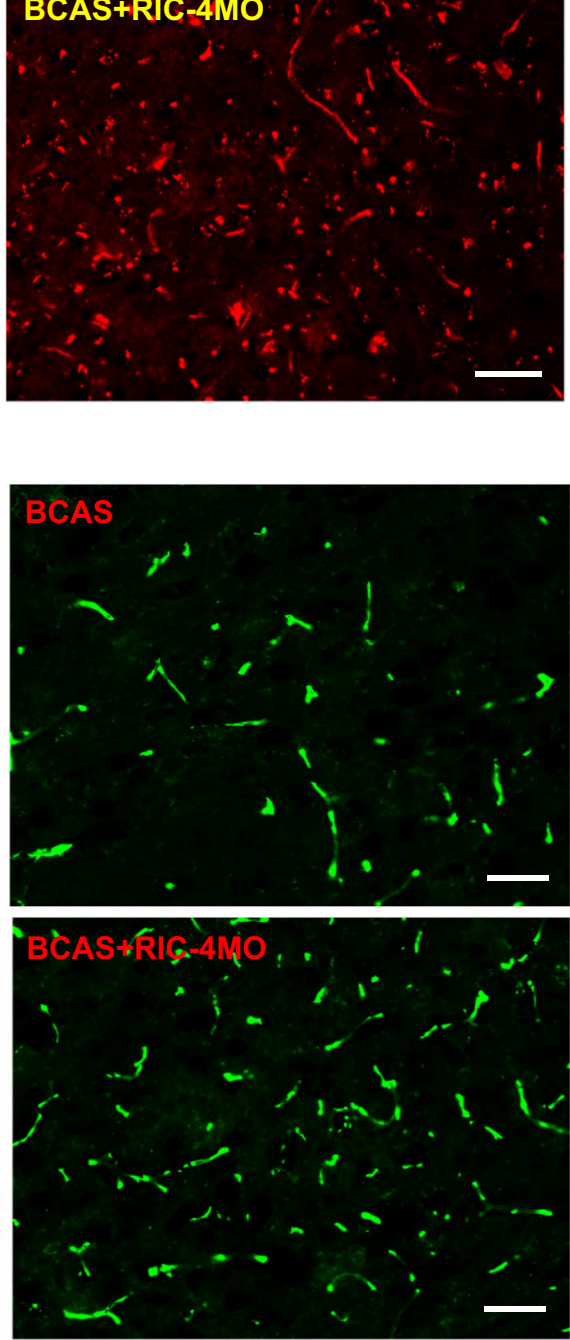
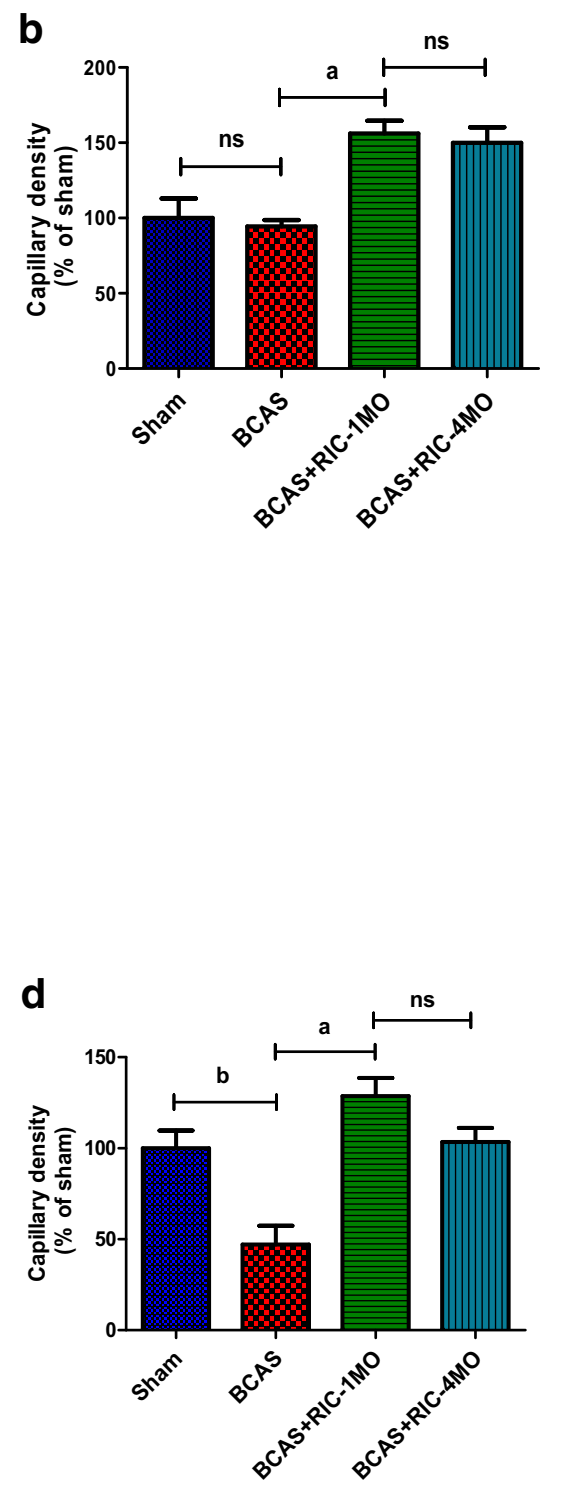

Fig. 4 RIC promotes angiogenesis and arteriogenesis. a, c Representative photomicrographs of single immunofluorescence for CD31 (red, (a)) and $\alpha$-SMA (green, (c)) or double immunofluorescence (Supplemental Figs. 4 and 5 for CD31 and $\alpha$-SMA with DAPI) for vessels in the striatum (caudoputamen) of each indicated group at 6 months with or without RIC therapy (scale bar for CD31 and $\alpha$-SMA $=20 \mu \mathrm{m}$ $20 \times)$. b, d The quantitative analysis shows of capillary density at 6 months in each indicated group ( $N=4$ to $6 /$ group; ${ }^{\mathrm{b}} p<0.01$ vs sham; ${ }^{\mathrm{a}} p<0.001$ vs BCAS+RIC-1M/4MO) from the flow cytometry studies showed a corresponding increase in the EPC count (as indicated by increased expression of CD31 and VEGF-R2) and in macrophage expression and polarization (as indicated by increased expression of CD11b, F4/80, and CD206) with RIC therapy (Fig. 6a, b).

RIC therapy for 3 weeks post BCAS significantly increased the plasma nitrite levels compared to BCAS 
a
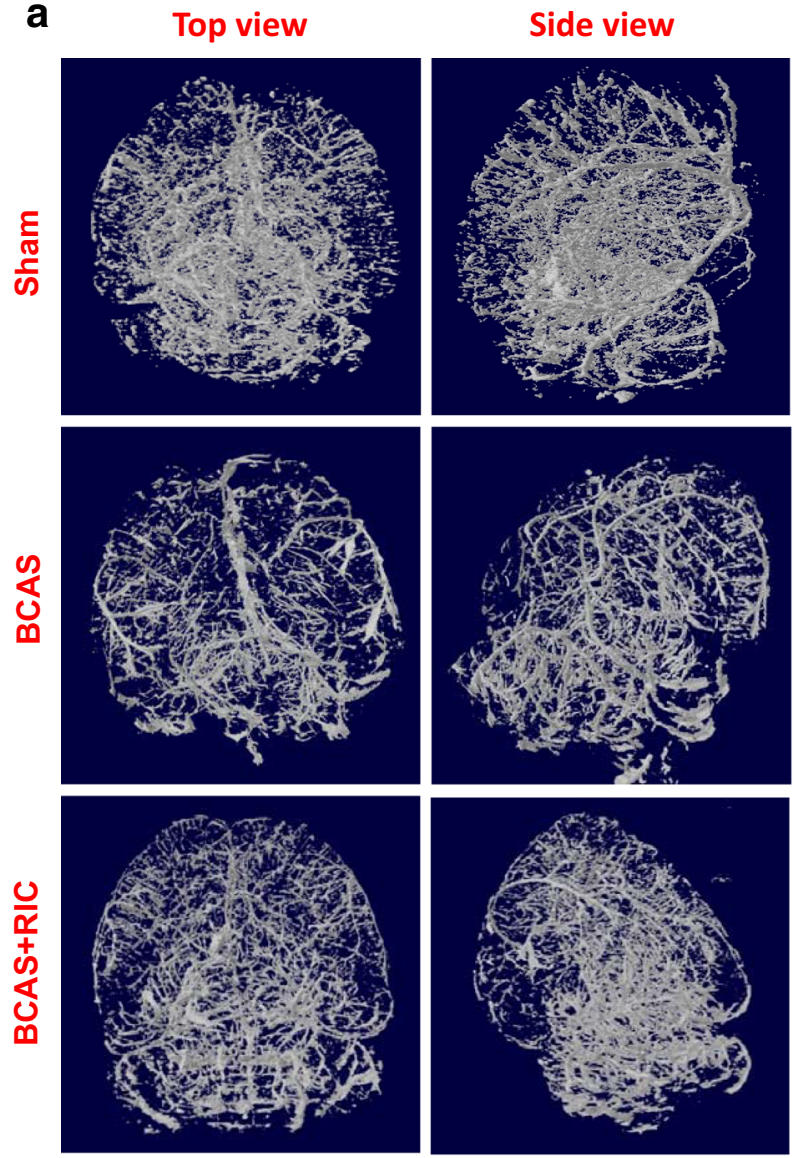

Fig. 5 RIC facilitates cerebrovascular angioarchitecture with 3 weeks treatment after BCAS. (a) Representative 3D images showing the whole cerebrovascular angioarchitecture from a top, side, and bottom view of sham, BCAS, and BCSA+RIC groups of mice brain.

without RIC. However, RIC therapy for 1-MO and 4MO showed a trend but insignificant increase in plasma nitrite levels at 6 months compared to the BCAS group (Fig. 6c, d). However, plasma nitrite level in the BCAS group was significantly decreased as compared to the sham group. Probably the short half-life of NO and long delay after RIC was finished caused NO levels to decrease.

\section{Discussion}

RIC can be performed before acute cerebral ischemia (preconditioning), during acute ischemia (per-conditioning), or after reperfusion (post conditioning). There is a large body of evidence that acute remote ischemic conditioning is a powerful cerebroprotectant in acute focal cerebral ischemia models $[27,28]$. The current study proposes C-RIC as a therapeutic paradigm in chronic mild ischemia. The repetitive use of RIC for weeks or b

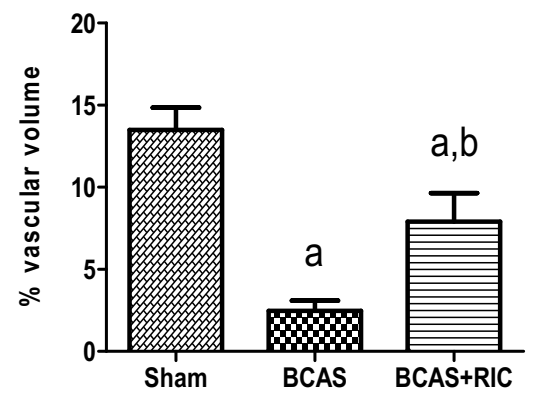

C

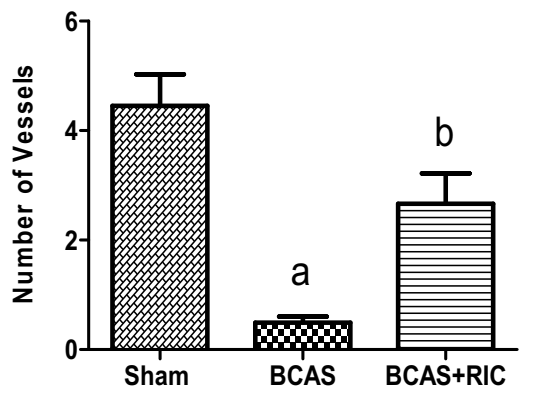

Histogram showing vascular volume percentage (b) and number of vessels (c) for linear space between vessels, density, and lumen thickness (Supplemental Fig. 9A-C). $N=8$ /group, ${ }^{a} p<0.01$ vs sham; ${ }^{\mathrm{b}} p<0.05$ vs BCAS

months can be an analogue to long-term daily exercise. There are fewer published studies and less data on CRIC in chronic cerebral ischemia. C-RIC administered for 6 months was effective in reducing recurrent stroke and TIA in two small randomized clinical trials in patients with intracranial atherosclerosis (ICAS) [29, 30]. There was increased CBF by SPECT in one trial suggesting that $\mathrm{C}$-RIC increased $\mathrm{CBF}$ in human patients. There is also a small clinical trial suggesting that C-RIC may reduce progression of white matter disease in patients with VCID. However, we have little preclinical data to support its use clinically, or to better understand its mechanisms. The BCAS mouse model has been offered as a model to test interventions in cerebral small vessel disease and VCID, and we now describe findings that support translation to the bedside.

First, our results show that both 1 month C-RIC (1$\mathrm{MO})$ and 4 months of C-RIC (4-MO) are equally effective in improving long-term (6 months) $\mathrm{CBF}$, working and spatial memory, and in improving balance and motor skills compared with sham animals. This is a crucial 


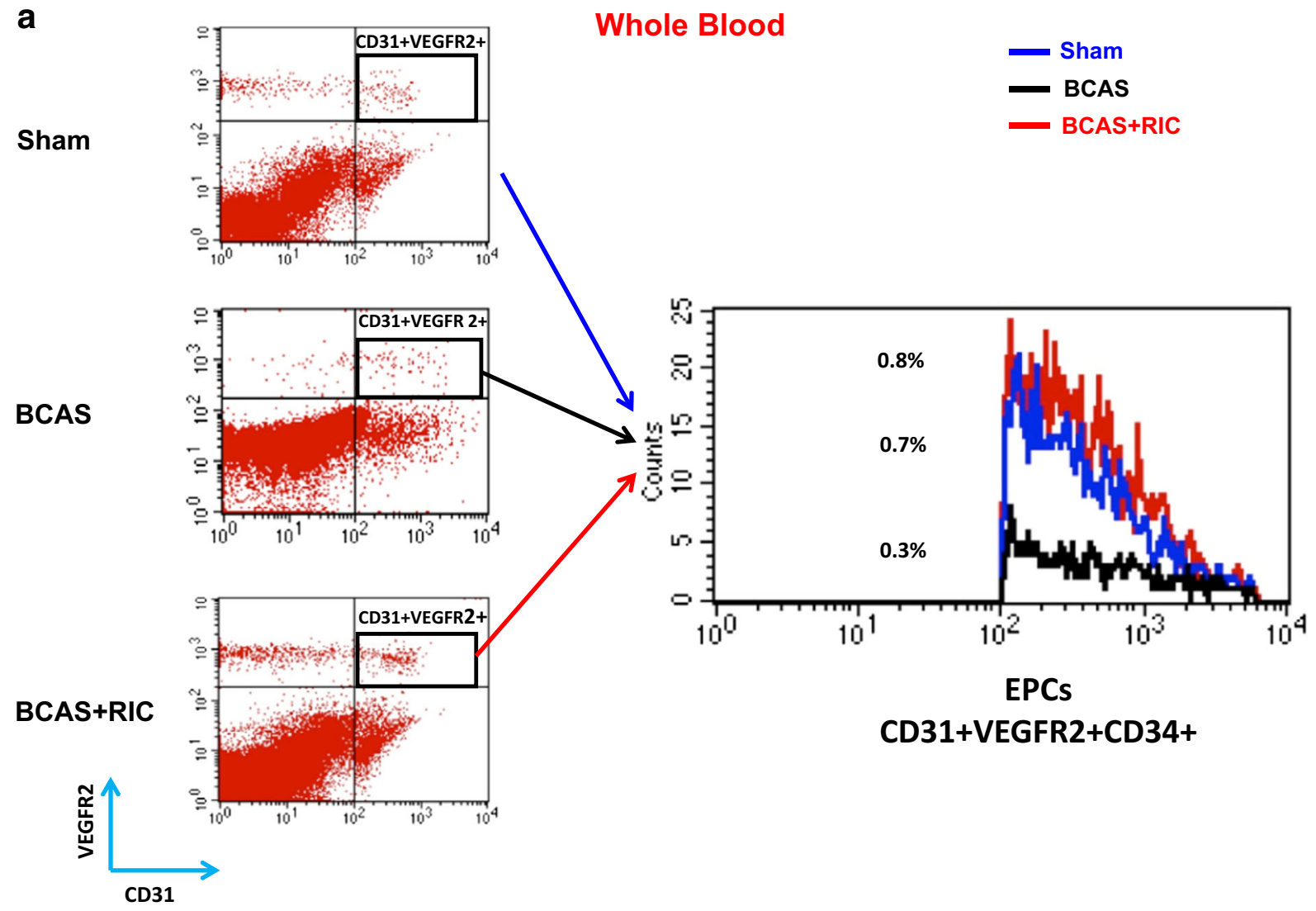

b

Whole Blood

Sham
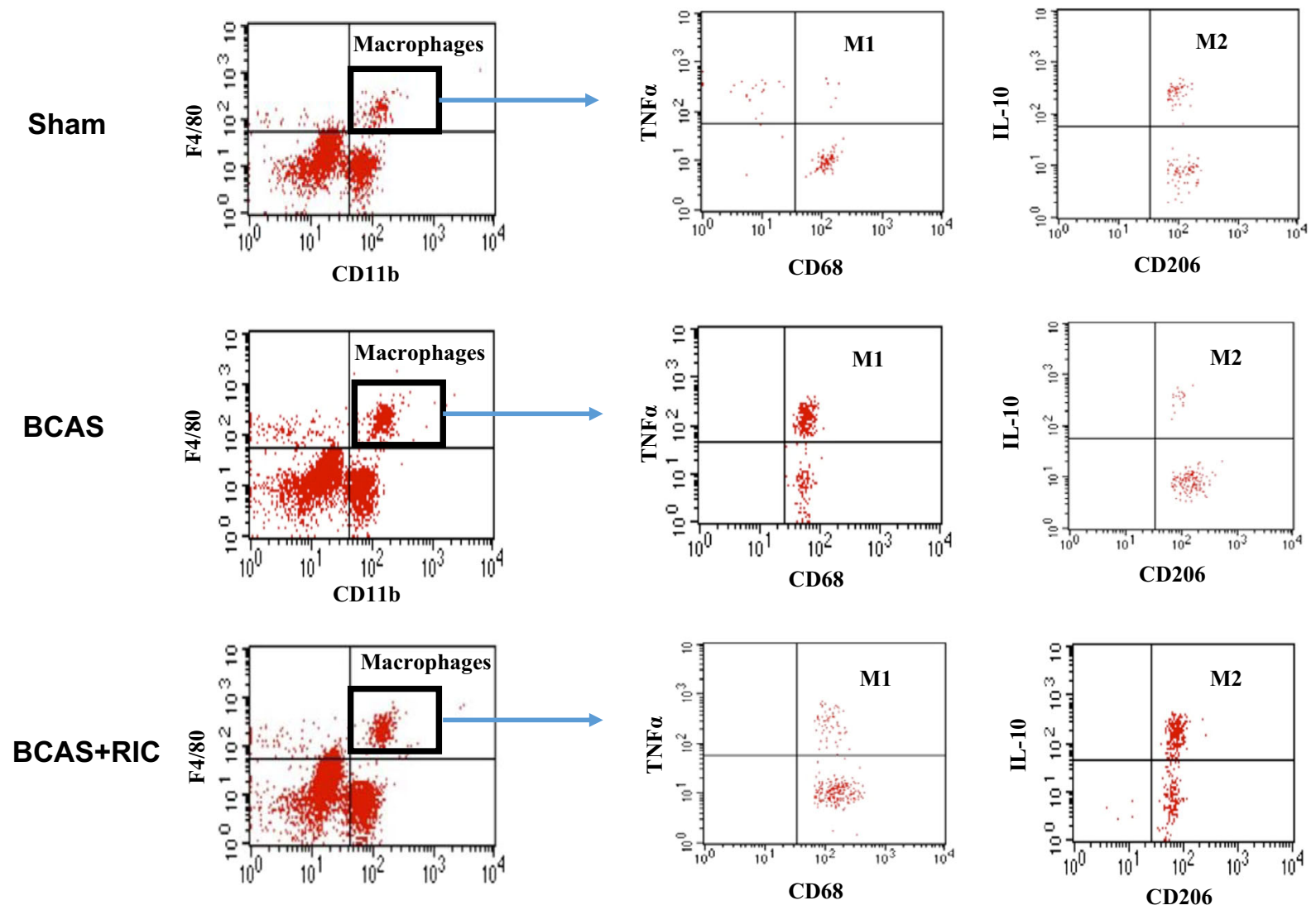

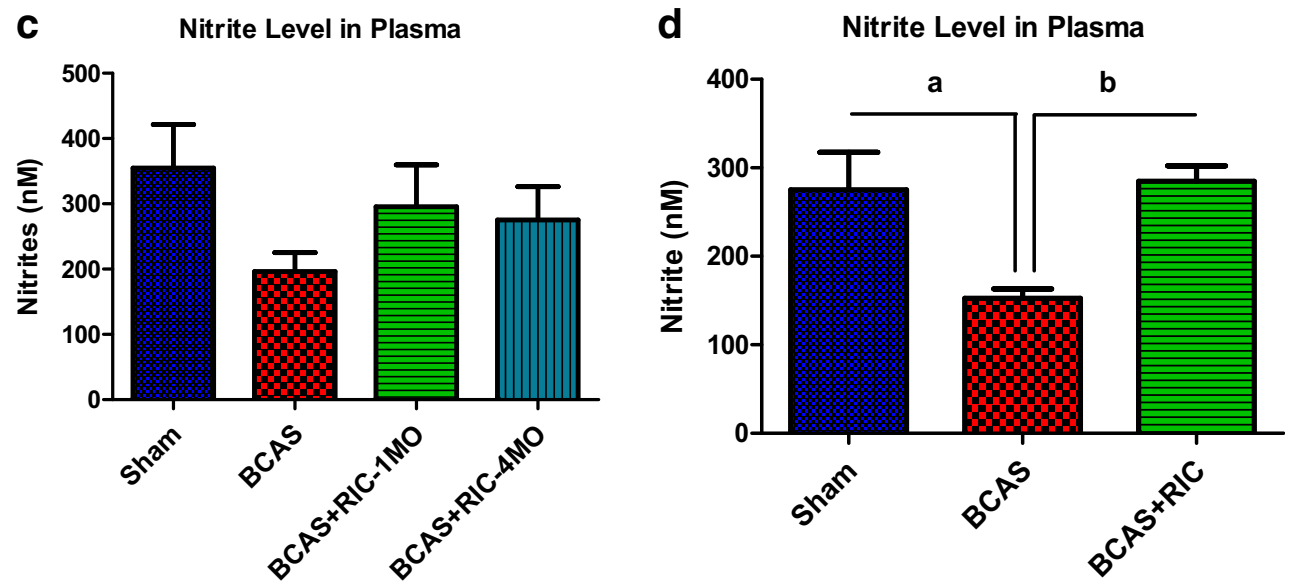

Fig. 6 continued.

piece of data suggesting that shorter term use of C-RIC (1 month) in patients with chronic ischemia may be as effective as longer term treatment (4 months). However, we exercise caution in extrapolating these findings to human VCID/small vessel disease, as the BCAS model has a known start and onset time. In VCID patients, the course of disease is insidious and there is no precise start time. Nevertheless, in our preclinical model, C-RIC appears to be efficient in improving the long-term $\mathrm{CBF}$ and long-term cognitive and motor function, likely by inducing angiogenesis and collateral remodeling. Our findings may have some implications to use C-RIC in ICAS where there is hypoperfusion, albeit from intracranial rather than extracranial stenosis. While the clinical trials in ICAS have used 6 months or 300 days of C-RIC, our results show that shorter durations may be effective.

Second, C-RIC for 3 weeks induces cerebral vascular remodeling, angiogenesis, arteriogenesis, and new collateral formation. These structural changes in the angioarchitecture may underlie the improvement in CBF. Beyond the functions of supplying oxygen and nutrients, endothelial cells have

Fig. 6 RIC therapy activates endothelial progenitor cells (EPCs) and increases M2/M1 macrophages in the blood 3 weeks treatment. (a) Flow cytometry graphs showing a significant increase the EPCs count, as indicated by increased expression of CD31, VEGFR2, and CD34. (b) Dropped CBF in BCAS group results in recruitment of macrophages in response to chronic ischemia. RIC therapy decreased the level of inflammatory M1 macrophages while it enhanced the level of anti-inflammatory M2 macrophages in the blood (as indicated by the expression of CD11b, F4/80; CD68, TNF $\alpha$; and CD206, IL-10). However, a high level of circulating M1 macrophage was counted in BCAS groups, indicating high inflammatory burden. RIC therapy activated circulating EPCs, thus reduced the vascular injury and protects ischemic brain. (c) RIC therapy with 1-MO or 4-MO showed a trend but insignificant increase in plasma nitrite levels at 6 months compared to BCAS group. However, RIC therapy for 3 weeks post BCAS significantly increased the plasma nitrite levels compared to BCAS sham RIC (d). Values are indicated as mean \pm SD. ${ }^{\mathrm{a}} p=0.0092$ vs sham; ${ }^{\mathrm{b}} p=0.0044$ vs BCAS+RIC trophic functions for neurons and oligodendrocytes. This angiogenesis and improved CBF are likely protective to oligodendrocytes and neurons. This increase in cerebral angiogenesis is similar to what is seen in the rodents' models of daily physical exercise for 3-4 weeks [31-34]. There are strong parallels between RIC and physical exercise. Both involve shear stress to the vasculature and upregulation of endothelial NOS (eNOS) and increased plasma nitrite [35, 36].

Third, similar to exercise, C-RIC increases circulating EPCs, which may be playing a role in angiogenesis. While there is controversy over whether EPCs incorporate directly into growing vessels or provide trophic effect to vessels, they are associated with angiogenesis and vascular health. Further studies are needed to define their participation in angiogenesis in our model.

Fourth, C-RIC is associated with increases in plasma nitrite, which suggests a role for increased NO bioavailability in mediating vascular remodeling. While once regarded as an inert molecule and oxidative end product of NO metabolism, research over the last decade has shown that nitrite serves a "storage" pool of NO derived from endogenous eNOS [37-39]. Nitrite circulates in the blood associated with RBC/hemoglobin and is reduced to $\mathrm{NO}$ in areas of hypoxemia, mediating hypoxic vasodilatation $[38,40]$. While NO is normally limited to a paracrine effect due to its very short half-life, the eNOS/nitrite/NO system provides a distant "endocrine" effect of NO. We showed in this study that increased NO bioavailability is associated with angiogenesis and collateral remodeling. Moreover, NO is associated with mobilization of endothelial progenitor cells.

We have demonstrated that C-RIC is effective in promoting angiogenesis and improving long-term functional outcome. The major risk factor for dementia and VCID is age. It is paramount to demonstrate these findings in aged mice of both sexes, and we are conducting series of these studies in our group. C-RIC is a safe and well-tolerated intervention that 
may be useful in patients with VCID. Further studies are needed to determine the dosing and to develop biomarkers. Both EPCs and plasma nitrite may serve as useful biomarkers.

\section{Compliance with Ethical Standards}

Funding This work was supported by the NIH/NINDS R21NS09060901A1. We are special thankful to Mr. Richard Goodman, Hatteras Instruments, Cary, $\mathrm{NC}$, in accepting a proposed design and making a multichannel non-invasive programmable remote ischemic conditioner and for a generous gift to our laboratory.

Conflict of Interest The authors declare that they have no conflict of interest.

Ethical Approval All applicable institutional and national guidelines for the care and use of animals were followed.

Open Access This article is distributed under the terms of the Creative Commons Attribution 4.0 International License (http:// creativecommons.org/licenses/by/4.0/), which permits unrestricted use, distribution, and reproduction in any medium, provided you give appropriate credit to the original author(s) and the source, provide a link to the Creative Commons license, and indicate if changes were made.

\section{References}

1. Iadecola C. The pathobiology of vascular dementia. Neuron. 2013;80(4):844-66. doi:10.1016/j.neuron.2013.10.008.

2. Snowdon DA, Greiner LH, Mortimer JA, Riley KP, Greiner PA, Markesbery WR. Brain infarction and the clinical expression of Alzheimer disease. The Nun Study. JAMA. 1997;277(10):813-7.

3. Snyder HM, Corriveau RA, Craft S, Faber JE, Greenberg SM, Knopman D, et al. Vascular contributions to cognitive impairment and dementia including Alzheimer's disease. Alzheimers Dement : J Alzheimers Assoc. 2015;11(6):710-7. doi:10.1016/j.jalz.2014. 10.008 .

4. Corriveau RA, Bosetti F, Emr M, Gladman JT, Koenig JI, Moy CS, et al. The science of vascular contributions to cognitive impairment and dementia (VCID): a framework for advancing research priorities in the cerebrovascular biology of cognitive decline. Cell Mol Neurobiol. 2016;36(2):281-8. doi:10.1007/s10571-016-0334-7.

5. Verdelho A, Madureira S, Ferro JM, Baezner H, Blahak C, Poggesi A, et al. Physical activity prevents progression for cognitive impairment and vascular dementia: results from the LADIS (Leukoaraiosis and Disability) study. Stroke. 2012;43(12):3331-5. doi:10.1161/STROKEAHA.112.661793.

6. O'Sullivan M, Lythgoe DJ, Pereira AC, Summers PE, Jarosz JM, Williams SC, et al. Patterns of cerebral blood flow reduction in patients with ischemic leukoaraiosis. Neurology. 2002;59(3):3216.

7. Bernbaum M, Menon BK, Fick G, Smith EE, Goyal M, Frayne R, et al. Reduced blood flow in normal white matter predicts development of leukoaraiosis. J Cereb Blood Flow Metab : Off J Int Soc Cereb Blood Flow Metab. 2015; doi:10.1038/jcbfm.2015.92.

8. Promjunyakul N, Lahna D, Kaye JA, Dodge HH, Erten-Lyons D, Rooney WD, et al. Characterizing the white matter hyperintensity penumbra with cerebral blood flow measures. NeuroImage Clin. 2015;8:224-9. doi:10.1016/j.nicl.2015.04.012.

9. Bink DI, Ritz K, Aronica E, van der Weerd L, Daemen MJ. Mouse models to study the effect of cardiovascular risk factors on brain structure and cognition. J Cereb Blood Flow Metab : Off J Int Soc
Cereb Blood Flow Metab. 2013;33(11):1666-84. doi:10.1038/ jcbfm.2013.140.

10. Shibata M, Ohtani R, Ihara M, Tomimoto H. White matter lesions and glial activation in a novel mouse model of chronic cerebral hypoperfusion. Stroke. 2004;35(11):2598-603. doi:10.1161/01. STR.0000143725.19053.60.

11. Shibata M, Yamasaki N, Miyakawa T, Kalaria RN, Fujita Y, Ohtani $\mathrm{R}$, et al. Selective impairment of working memory in a mouse model of chronic cerebral hypoperfusion. Stroke. 2007;38(10):2826-32. doi:10.1161/strokeaha.107.490151.

12. Holland PR, Searcy JL, Salvadores N, Scullion G, Chen G, Lawson $\mathrm{G}$, et al. Gliovascular disruption and cognitive deficits in a mouse model with features of small vessel disease. J Cereb Blood Flow Metab : Off J Int Soc Cereb Blood Flow Metab. 2015;35(6):100514. doi: $10.1038 / \mathrm{jcbfm} .2015 .12$.

13. Michelsen MM, Stottrup NB, Schmidt MR, Lofgren B, Jensen RV, Tropak M, et al. Exercise-induced cardioprotection is mediated by a bloodborne, transferable factor. Basic Res Cardiol. 2012;107(3): 260. doi:10.1007/s00395-012-0260-x.

14. Hess DC, Blauenfeldt RA, Andersen G, Hougaard KD, Hoda MN, Ding Y, et al. Remote ischaemic conditioning-a new paradigm of self-protection in the brain. Nat Rev Neurol. 2015;11(12):698-710. doi:10.1038/nrneurol.2015.223.

15. Khan MB, Hoda MN, Vaibhav K, Giri S, Wang P, Waller JL, et al. Remote ischemic postconditioning: harnessing endogenous protection in a murine model of vascular cognitive impairment. Transl Stroke Res. 2014; doi:10.1007/s12975-014-0374-6.

16. Fisher M, Feuerstein G, Howells DW, Hurn PD, Kent TA, Savitz SI, et al. Update of the stroke therapy academic industry roundtable preclinical recommendations. Stroke. 2009;40(6):2244-50. doi:10. 1161/STROKEAHA.108.541128.

17. Lapchak PA, Zhang JH, Noble-Haeusslein LJ. RIGOR guidelines: escalating STAIR and STEPS for effective translational research. Transl Stroke Res. 2013;4(3):279-85. doi:10.1007/s12975-0120209-2.

18. Ropcke DM, Hjortdal VE, Toft GE, Jensen MO, Kristensen SD. Remote ischemic preconditioning reduces thrombus formation in the rat. J Thromb haemost : JTH. 2012;10(11):2405-6. doi:10.1111/ j.1538-7836.2012.04914.x.

19. Yuan HJ, Zhu XH, Luo Q, Wu YN, Kang Y, Jiao JJ, et al. Noninvasive delayed limb ischemic preconditioning in rats increases antioxidant activities in cerebral tissue during severe ischemia-reperfusion injury. J Surg Res. 2012;174(1):176-83. doi:10.1016/j.jss.2010.11.001.

20. Fulton D, Harris MB, Kemp BE, Venema RC, Marrero MB, Stepp DW. Insulin resistance does not diminish eNOS expression, phosphorylation, or binding to HSP-90. Am J Physiol Heart Circ Physiol. 2004;287(6):H2384-93. doi:10.1152/ajpheart.00280. 2004.

21. Wakade C, Sukumari-Ramesh S, Laird MD, Dhandapani KM, Vender JR. Delayed reduction in hippocampal postsynaptic density protein-95 expression temporally correlates with cognitive dysfunction following controlled cortical impact in mice. J Neurosurg. 2010;113(6):1195-201. doi:10.3171/2010.3.JNS091212.

22. Li Y, Kim J. CB2 cannabinoid receptor knockout in mice impairs contextual long-term memory and enhances spatial working memory. Neural Plast. 2016;2016:9817089. doi:10.1155/2016/9817089.

23. Luong TN, Carlisle HJ, Southwell A, Patterson PH. Assessment of motor balance and coordination in mice using the balance beam. $\mathrm{J}$ Vis Exp. 2011;49 doi:10.3791/2376.

24. Klein SM, Vykoukal J, Lechler P, Zeitler K, Gehmert S, Schreml S, et al. Noninvasive in vivo assessment of muscle impairment in the mdx mouse model - a comparison of two common wire hanging methods with two different results. J Neurosci Methods. 2012;203(2):292-7. doi:10.1016/j.jneumeth.2011.10.001. 
25. Baban B, Hansen AM, Chandler PR, Manlapat A, Bingaman A, Kahler DJ, et al. A minor population of splenic dendritic cells expressing CD19 mediates IDO-dependent T cell suppression via type I IFN signaling following B7 ligation. Int Immunol. 2005;17(7): 909-19. doi:10.1093/intimm/dxh271.

26. Baban B, Liu JY, Mozaffari MS. Pressure overload regulates expression of cytokines, gammaH2AX, and growth arrest- and DNAdamage inducible protein 153 via glycogen synthase kinase-3beta in ischemic-reperfused hearts. Hypertension. 2013;61(1):95-104. doi:10.1161/HYPERTENSIONAHA.111.00028

27. Hess DC, Hoda MN, Bhatia K. Remote limb perconditioning [corrected] and postconditioning: will it translate into a promising treatment for acute stroke? Stroke. 2013;44(4):1191-7. doi:10. 1161/STROKEAHA.112.678482.

28. Hoda MN, Siddiqui S, Herberg S, Periyasamy-Thandavan S, Bhatia $\mathrm{K}$, Hafez SS, et al. Remote ischemic perconditioning is effective alone and in combination with intravenous tissue-type plasminogen activator in murine model of embolic stroke. Stroke. 2012;43(10): 2794-9. doi:10.1161/STROKEAHA.112.660373.

29. Meng R, Asmaro K, Meng L, Liu Y, Ma C, Xi C, et al. Upper limb ischemic preconditioning prevents recurrent stroke in intracranial arterial stenosis. Neurology. 2012;79(18):1853-61. doi:10.1212/ WNL.0b013e318271f76a.

30. Meng R, Ding Y, Asmaro K, Brogan D, Meng L, Sui M, et al. Ischemic conditioning is safe and effective for octo- and nonagenarians in stroke prevention and treatment. Neurotherapeutics. 2015; doi:10.1007/s13311-015-0358-6.

31. Endres M, Gertz K, Lindauer U, Katchanov J, Schultze J, Schrock $\mathrm{H}$, et al. Mechanisms of stroke protection by physical activity. Ann Neurol. 2003;54(5):582-90. doi:10.1002/ana.10722.

32. Ding Y, Li J, Luan X, Ding YH, Lai Q, Rafols JA, et al. Exercise pre-conditioning reduces brain damage in ischemic rats that may be associated with regional angiogenesis and cellular overexpression of neurotrophin. Neuroscience. 2004;124(3):583-91. doi:10.1016/ j.neuroscience.2003.12.029.

33. Ding YH, Luan XD, Li J, Rafols JA, Guthinkonda M, Diaz FG, et al. Exercise-induced overexpression of angiogenic factors and reduction of ischemia/reperfusion injury in stroke. Curr Neurovasc Res. 2004;1(5):411-20.

34. Ding YH, Young CN, Luan X, Li J, Rafols JA, Clark JC, et al. Exercise preconditioning ameliorates inflammatory injury in ischemic rats during reperfusion. Acta Neuropathol. 2005;109(3):23746. doi:10.1007/s00401-004-0943-y.

35. Calvert JW, Condit ME, Aragon JP, Nicholson CK, Moody BF, Hood RL, et al. Exercise protects against myocardial ischemiareperfusion injury via stimulation of beta(3)-adrenergic receptors and increased nitric oxide signaling: role of nitrite and nitrosothiols. Circ Res. 2011;108(12):1448-58. doi:10.1161/CIRCRESAHA. 111.241117.

36. Barbosa VA, Luciano TF, Marques SO, Vitto MF, Souza DR, Silva LA, et al. Acute exercise induce endothelial nitric oxide synthase phosphorylation via Akt and AMP-activated protein kinase in aorta of rats: role of reactive oxygen species. Int J Cardiol. 2013;167(6): 2983-8. doi:10.1016/j.ijcard.2012.08.050.

37. Gladwin MT, Tejero J. Nitrite-NO bailout for a NOS complex too big to fail. Nat Med. 2011;17(12):1556-7. doi:10.1038/nm.2591.

38. Kim-Shapiro DB, Gladwin MT. Mechanisms of nitrite bioactivation. Nitric Oxide : Biol Chem / Off J Nitric Oxide Soc. 2014;38:58-68. doi:10.1016/j.niox.2013.11.002.

39. Lundberg JO, Gladwin MT, Weitzberg E. Strategies to increase nitric oxide signalling in cardiovascular disease. Nat Rev Drug Discov. 2015;14(9):623-41. doi:10.1038/nrd4623.

40. Liu C, Wajih N, Liu X, Basu S, Janes J, Marvel M, et al. Mechanisms of human erythrocytic bioactivation of nitrite. J Biol Chem. 2015;290(2):1281-94. doi:10.1074/jbc.M114.609222. 